\title{
200W Gain-Switched-Diode-Seeded, Single-Polarization, Narrow-Linewidth, All-Fiber, Picosecond MOPA
}

\author{
Peh Siong Teh, Ho-Yin Chan, Richard J. Lewis, David P. Shepherd, Shaif-ul Alam and David J. Richardson \\ Optoelectronics Research Centre, University of Southampton, Southampton SO17 1BJ, UK \\ pst1r09@orc.soton.ac.uk
}

\begin{abstract}
We report a fully fiberized, single-polarization, gain-switched, diode-seeded fiber MOPA delivering $28 \mathrm{ps}$ pulses at a repetition frequency of $214 \mathrm{MHz}$ with $200 \mathrm{~W}$ of average output power and up to $0.93 \mu \mathrm{J}$ pulse energy.
\end{abstract}

OCIS codes: (060.2320) Fiber Optics Amplifiers and Oscillators; (320.5390) Picosecond phenomena; (140.5960)

Semiconductor lasers

\section{Introduction}

High-power lasers operating in the picosecond regime are currently in demand for a number of applications including laser machining, material processing and frequency-doubling. Ti:sapphire lasers are commonly used for these applications, but recent demonstrations of fiberized master-oscillator-power-amplifier (MOPA) systems provide an alternative solution and are capable of operating at much higher average power levels. Up to $157 \mathrm{~W}$ of output power was demonstrated recently using a mode-locked laser seeded MOPA [1], whilst a gain-switched laserdiode-seeded MOPA produced an output power in the region of 100W [2]. It is worth noting that such high average power sources combined offering high repetition rates and single polarization output are becoming increasingly interesting for the pumping of optical parametric oscillators (OPOs) [3].

Herein we report a fully fiberized gain-switched laser-diode-seeded MOPA system generating linearly polarized, $28 \mathrm{ps}$ pulses at a repetition frequency of $214 \mathrm{MHz}$ with a maximum average output power of $200 \mathrm{~W}$.

\section{Experimental Setup}

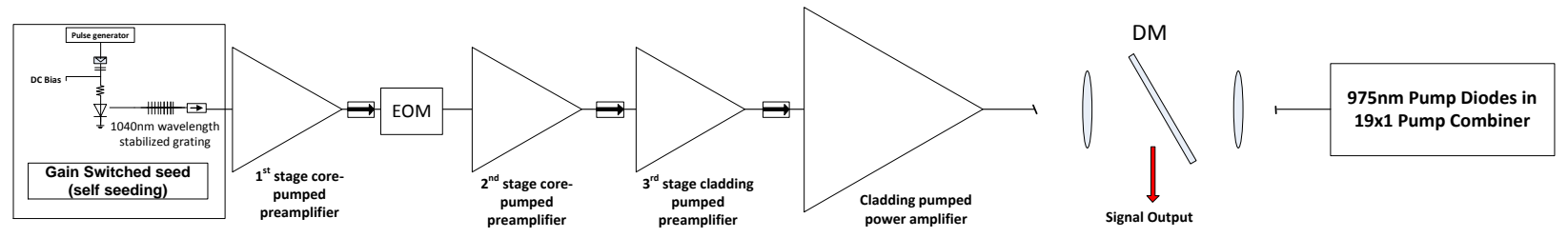

Figure 1: Fully fiberized picosecond seed laser based YDFA MOPA system incorporating 4 amplifier stages.

A schematic of our experimental setup is shown in Figure 1. A 1040nm Fabry Perot laser diode (3SPhotonics) with a polarization maintaining (PM) fiber pigtail was gain-switched using a stable train of sinusoidal electrical current pulses superimposed upon a DC bias. The pigtail of the semiconductor laser diode (SLD) was spliced to a PM fiber Bragg grating (FBG) with a $3 \mathrm{~dB}$ bandwidth of $0.24 \mathrm{~nm}$ and a reflectivity of $7.2 \%$. The repetition frequency was tuned to $858 \mathrm{MHz}$ to achieve synchronization between the emitted pulses from the diode and reflected pulses from the FBG. The measured side-mode suppression ratio (SMSR) was $\sim 40 \mathrm{~dB}$ with a polarization extinction ratio (PER) of over 20dB. This gain switching technique, whereby a uniform FBG has been used for the self-seeding mechanism, produced near-transform-limited pulses (time-bandwidth product estimated to be 0.53 assuming Gaussian-shaped pulses) with a FWHM of $\sim 28 \mathrm{ps}$ and a pulse energy of $7 \mathrm{pJ}$. The pulses were then amplified in a four-stage PM ytterbium-doped fiber amplifier (YDFA) MOPA chain.

The first stage amplifier consisted of a 50cm-long core-pumped YDFA $(5 \mu \mathrm{m}$ core and $130 \mu \mathrm{m}$ cladding diameter) pumped by a $180 \mathrm{~mW}$ single-mode $976 \mathrm{~nm}$ pigtailed laser diode. An average output power of $50 \mathrm{~mW}$ was obtained from this stage. It was then coupled into an inline electro-optic modulator (EOM), which was operated as a pulse picker in order to reduce the pulse repetition frequency. An average power of $4 \mathrm{~mW}$ was measured at the output of the EOM when the operating frequency was brought down to $214 \mathrm{MHz}$. Due to the excess loss of the EOM, a second core-pumped YDFA with an active fiber length of $1 \mathrm{~m}$ was used to ensure adequate seeding of the cladding-pumped third-stage amplifier. The maximum output power from this second-stage amplifier was $20 \mathrm{~mW}$. 
The third-stage YDFA comprised a 2.5m-long cladding-pumped large-mode-area (LMA) fiber with a core diameter of $10 \mu \mathrm{m}, 0.08 \mathrm{NA}$ and an inner-cladding diameter of $125 \mu \mathrm{m}, 0.46 \mathrm{NA}$. The fiber was co-directionally pumped by a $10 \mathrm{~W}, 975 \mathrm{~nm}$ multi-mode (MM) pump diode through the use of a fiberized $(2+1) \times 1 \mathrm{MM}$ pump combiner. A maximum average output power of $2.6 \mathrm{~W}$ was obtained from this stage. The output was then taperspliced to the final-stage amplifier, comprised of a $2.6 \mathrm{~m}$-long LMA fiber with core and cladding diameters/NA of $30 \mu \mathrm{m} / 0.06$ and $250 \mu \mathrm{m} / 0.46$ respectively (NUFERN PLMA-YDF-30/250-VIII). The tapered splice ensured singlemode operation whilst the use of a fast-axis blocking PM isolator ensured single-polarization seeding to the finalstage amplifier. The fiber was counter-pumped via free-space coupling. A dichroic mirror (DM) was used (as shown in Fig. 1) to separate pump and signal beams. An end cap was spliced to the output of the LMA fiber to avoid damage to the end facet.

\section{Results}
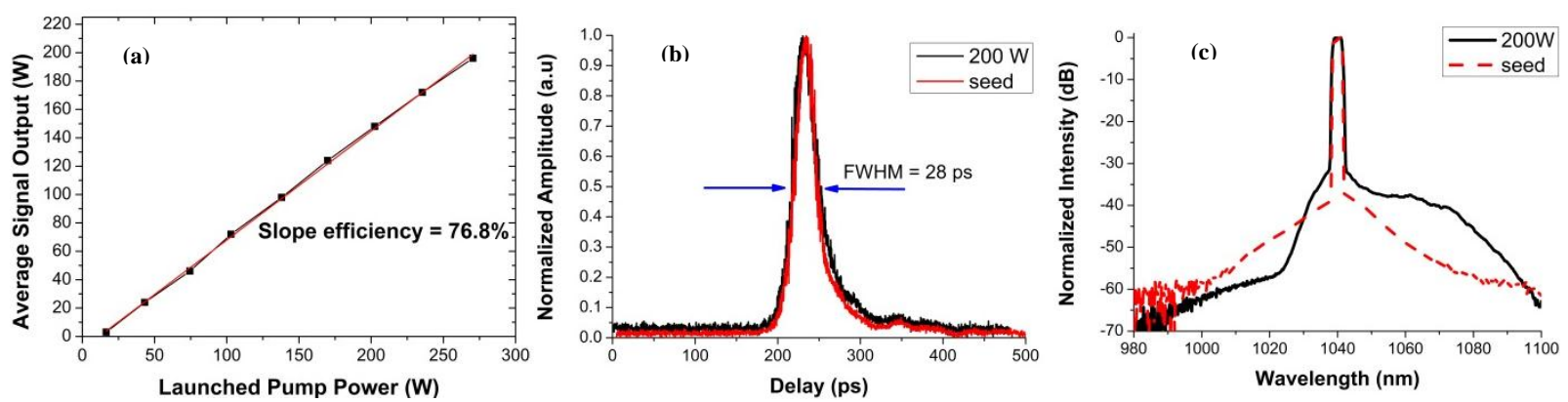

Figure 2(a) Overall slope efficiency at $214 \mathrm{MHz}$, (b) Pulse shapes measured with 32GHz photo-detector, (c) Spectra measured with 2nm resolution with center wavelength at $1040 \mathrm{~nm}$.

A maximum output power of $200 \mathrm{~W}$ was obtained with a total launched pump power of $270 \mathrm{~W}$ as shown in Figure 2(a), corresponding to a slope efficiency of $76.8 \%$ (around $90 \%$ of the launched pump power was effectively coupled into the active fiber). A pulse energy of $0.93 \mu \mathrm{J}$ and a peak power of $33 \mathrm{~kW}$ were obtained at a pulse repetition frequency (PRF) of 214MHz. The total end-to-end gain achieved in this system was $\sim 51 \mathrm{~dB}$. The measured PER was more than $13 \mathrm{~dB}$ while the $\mathrm{M}^{2}$ value was measured to be $\sim 1.1$. As shown in Figure 2(b), no significant temporal distortion was observed even at the maximum output power. The full width at half maximum of $\sim 28 \mathrm{ps}$ at 200W was directly measured with a wideband photo-detector and oscilloscope. Figure 2(c) compares the spectra before and after the fiber MOPA chain. The amplified spontaneous emission (ASE) is suppressed by more than $30 \mathrm{~dB}$ below the signal peak at the maximum operating output power of $200 \mathrm{~W}$. With such a high optical signal-tonoise ratio (OSNR), further power scaling is possible; the only limitation on maximum peak power being the introduction of nonlinear effects, especially stimulated Raman scattering (SRS). Further details on signal amplification at lower PRF will be presented at the meeting.

\section{Conclusion}

We have demonstrated a 200W, single polarization, 28 ps pulse source, based on a gain-switched, diode-seeded, allfiber MOPA. A pulse energy of $0.93 \mu \mathrm{J}$ and a peak power of $33 \mathrm{~kW}$ were obtained. The experimental results show that further power scaling and pulse-energy extraction will be possible in due course from this system.

\section{References}

[1] Rui Song, Jing Hou, Shengping Chen, Weiqiang Yang, and Qisheng Lu, "157 W all fiber high power picosecond laser,” Appl. Opt., 2012. 51(13): p. 2497-2500

[2] K. K. Chen, J. H. V. Price, S.-U. Alam, J. R. Hayes, D. J. Lin, A. Malinowski, and D. J. Richardson, "Polarisation maintaining 100W Yb-fiber MOPA producing $\mu \mathrm{J}$ pulses tunable in duration from 1 to 21 ps," Opt. Express18(14), 14385-14394 (2010).

[3] F. Kienle, P. S. Teh, D. Lin, S.-U. Alam, J. H. V. Price, D. C. Hanna, D. J. Richardson, and D. P. Shepherd, "High-power, high repetitionrate, green-pumped, picosecond LBO optical parametric oscillator," Opt. Express20(7), 7008-7014 (2012). 